\title{
Die periglazialen Strukturbodenbildungen als Folgen der Hydratationsvorgänge im Boden
}

\author{
Von Erwin Schenk, Gießen \\ Mit 7 Abb. im Text
}

$\mathrm{Zus}$ ammenf assung. Die Strukturbildungen im Frostboden werden als Auswirkungen der Hydratationsvorgänge im gefrierenden Boden gedeutet. Die das Wasseraufnahmevermögen der Bodenteilchen übersteigende Wasserzufuhr beim Gefrieren ergibt sich aus den Energiedifferenzen der hydratisierenden Teilchen. Angetrieben wird die Wasserbewegung von dem elektrischen Potential der wachsenden Eiskristalle, das beträchtlich höher ist als das der übrigen Komponenten des gefrierenden polydispersen Systems Boden.

A bstract. The froststructures in soils are explained to be effects of the processes of hydratation. The water supply and water movement is caused by the differences in osmotic pressure, which results from the diëlectrical properties of the components of the polydisperse system soil.

\section{N H A L T}

1. Einführung

2. Quellung und Konvektion

3. Zustandsformen und Bewegungsvorgänge

4. Der Frostboden als kolloidales System

5. Hydratation

6. Dehydratation und Eiskeilbildung

7. Horizontale Eisschichten und vertikale Wasserbewegung

8. Frosteindringung

9. Frosthebung

10. Lentokapillarer Punkt

11. Rückblick

12. Zusammenfassung

Literaturverzeichnis.

\section{E in $f \ddot{u} h \mathrm{r} u \mathrm{ng}$}

Die große Zahl der Theorien, die zur Erklärung der Phänomene der Strukturbodenbildungen entwickelt worden sind, und die Tatsache, daß auch heute noch Erklärungen gegeben werden, die gesicherten Erkenntnissen der Strukturbodenforschung nicht entsprechen, zeigt eindringlich, daß bislang keine befriedigende Lösung gefunden worden ist. Wohl hat sich die Zahl der Theorien durch die fortschreitenden Erkenntnisse von selbst gemindert und auf 2 oder 3 im Grundsätzlichen auseinandergehende Erklärungen reduziert; doch bestehen im Detail noch beträchtliche Meinungsverschiedenheiten und dabei ein unklares Nebeneinander von verschiedenartigen Prinzipien der Mechanik und Dynamik.

Einerseits sind es die Kräfte kolloidchemischer Art, andererseits die Gesetze der reinen Mechanik, die antreibend und formend wirken sollen. Von den Faktoren Frostdruck, Frostschub und Frosthebung, Solifluktion im eigentlichen (R. SERnander 1905, J. G. Andersson 1906, O. Nordenskjöld 1909, B. Högвom 1908/09) und erweiterten Sinn (C. Troll 1944, 1948), Kontraktion, Quellung und Konvektion, Erosion und Windwirkung stehen bei den einzelnen Beobachtern und Forschern bald diese, bald jene im Vordergrund bei der Erklärung der Bildungsumstände und der Mechanik, ohne daß dabei auf die Grundgesetze der Frostwirkung im Boden als einem polydispersen System konsequent aufgebaut worden ist.

Eine allererste Voraussetzung für eine zutreffende Erklärung sind aber nun eindeutig klare und sichere Erkenntnisse in der Mechanik der Froststrukturböden, d. h. eine genaue Trennung, Gliederung und Folge der $\mathrm{k}$ in e mat is c he n Abläufe und der 
dy $\mathrm{n}$ a $\mathrm{m}$ is che $\mathrm{n}$ Antriebe. Sie wiederum kann nur aufgebaut werden, wenn die $\mathrm{Sta}$ tik des Bodens bzw. des Wassers im Boden bei Frosteinwirkung klar erkannt ist. Die Frostbodenforschung der Straßenbauer hat viele sichere Ergebnisse gebracht und nicht nur manche Beziehungen zwischen Korngröße und Frosthebung aufgedeckt, sondern auch zu ziemlich klaren zahlenmäßigen Formulierungen geführt. Die Frage aber, warum die Korngrößen von entscheidender Bedeutung sind, blieb bis heute unbefriedigend beantwortet, zumal die Kräfte der Kapillarität, auf der die bisherigen Erklärungen mehr oder weniger aufbauen, nicht mehr als maßgebend angesehen werden können, seit P. VAgeler (1932) ihre Problematik herausgestellt hat (s. a. Alten \& Kurmies 1939). Ebensowenig konnte nach den bisherigen Theorien die Bildung der Eiskeilnetze und der Eiskeile überhaupt und die Frage nach der Dimensionierung der Strukturen geklärt werden. Verfasser hat versucht, eine solche Mechanik herauszuarbeiten, nach der grundsätzlich alle Strukturböden zwanglos verständlich werden (E. Schenk 1955). Hierbei wurden bereits die Kräf te und Vorgänge der H ydrat a tion soweit herangezogen, wie es für das Verständnis der Mechanik erforderlich war. Der Boden wurde dabei nach dem Gesichtspunkt der modernen Bodenkunde als polydisperses System von Wasser, Bodenteilchen und Luft berïcksichtigt, d. h. also der kolloidchemische Zustand des Bodens. Aufgabe zukünftiger Forschung wird es sein, experimentell von hier aus weiterzuarbeiten.

\section{Quellung und Konvektion}

Bisher sind nur 2 Versuche gemacht worden, die Phänomene und Probleme der Froststrukturböden unter Berücksichtigung des kolloidchemischen Charakters des Bodens zu lösen. Der eine Versuch wurde von H. STECHE unternommen, der andere von $\mathrm{K}$. GRIPP und von ihm bis in alle Konsequenzen durchgeführt.

H. Steche (1933) ging von dem Gedanken aus, daß die Kräfte der Quellung, Dispersion und Koagulation des kolloidal-dispersen Systems maßgeblich strukturbildend und formend wirken. Dem lag die Vorstellung zu Grunde, daß die Bildung der gewölbten Formen im Frühjahr vor sich gehe, eine Folge der Wasseraufnahme sei (H. Steche S. 231) und daher durch Quellung der Bodenkolloide erklärt werden könne. Erst in der nachfolgenden Zeit mehrten sich die Berichte, aus denen hervorging, daß die Aufwölbungsformen mit dem Frost verbunden sind. Aber eine einheitliche Auffassung wurde in Anbetracht des höchst mannigfaltigen Nebeneinanders von gewölbten, flachen und eingesenkten Strukturformen doch nicht gewonnen. In Spitzbergen glaube ich erkannt zu haben, daß Formen mit flachen und gewölbten Oberflächen in der Auftauzeit bereits fossil oder halbfossil, jedenfalls äußerst träge sind. Vielfach sind es die eindrucksvollsten Strukturen. Meine darauf sich gründende Einteilung in aktive und inaktive Strukturen, lebendige einerseits und sehr träge und fossile andererseits (E. SCHENK 1955), dürfte Klarheit darüber schaffen, daß die Hüg elbild ungen allein ein Werkdes Frostes sind und daß Hügelformen, die die A uftauzeit überdauern, Kennzeichen für inaktive Strukturen sind. Danach ist es m. E. nicht mehr möglich, der Quellung ein wesentliches Moment bei der Strukturbildung zuzubilligen. Wir sehen also, wie die Kenntnis des tatsächlichen Ablaufes der Mechanik der Strukturen eine unerläßliche Voraussetzung für alle weiteren Stukturbodenforschungen ist. Die Rolle der Quellung, d. h. die Wasseraufnahme durch die Bodenteilchen, darf aber nun keineswegs ausgeschaltet oder auch nur unterschätzt werden. Sie hat tatsächlich eine große Bedeutung, wenn auch in einem anderen Sinne als ihn H. STECHE hervorhebt.

Die Kräfte der Konvektion sind zuerst von O. NordenskjöLd (1907), dann von A. Low (1925), H. Brunt (1925), K. Gripp (1926, 1935 und 1952), Y. Hudina (1933), S. Fujiwhara (1928) und V. Romanowski (1939) zur Erklärung der Strukturboden- 
phänomene herangezogen worden. Man stellte sich dabei vor, daß in der sehr stark mit Wasser aufgeschwemmten Erde infolge der sicherlich vorhandenen Temperaturunterschiede zwischen Oberfläche und Unterfläche des Auftaubodens und dadurch hervorgerufener Dichteunterschiede eine instabile Schichtung des Bodens bestehe, die durch brodelnde, kreisende Bewegungen in den Strukturen, den „Brodelkesseln“, einem Ausgleich, d. h. einem Gleichgewichtszustand zustreben würde. Dabei sollen Steinchen und grobe Bodenteilchen mitbewegt, aussortiert und an den Rändern der Strukturen angereichert werden können. K. GRIPP (1926, 1935 und 1952), der experimentell solche Strukturen erzeugte, denkt dabei auch an Absorptionskräfte des Lehms, berücksichtigt also sehr wohl eine Hydratation der Bodenteilchen. Ausgangspunkt der ganzen Uberlegungen und Versuche war also sehr richtig der Boden als polydisperses System von Wasser und Bodenteilchen und auch von Luft. Ebenso richtig erscheint die Berücksichtigung von Temperaturdifferenzen und Dichteunterschieden und schließlich die Vorstellung, daß sie im Strukturkessel einem Gleichgewicht zustreben müssen und daß dies nicht ohne Bewegungsvorgänge erfolgen kann. Diese Grundvorstellungen brauchen nicht aufgegeben zu werden, sie treffen auch heute noch zu. Doch kann ihnen nur eine Bedeutung als integrierende, zusätzliche, aber nicht entscheidende Faktoren zugemessen werden (E. SCHENK 1955). Nicht präzis genug aber scheinen oft noch die Vorstellungen um die Zustandsformen zu sein. Soweit nun Quellungs-, Konvektions- und all die anderen Theorien bei der Erklärung der Strukturphänomene von unzutreffenden $\mathrm{Zu}$ -

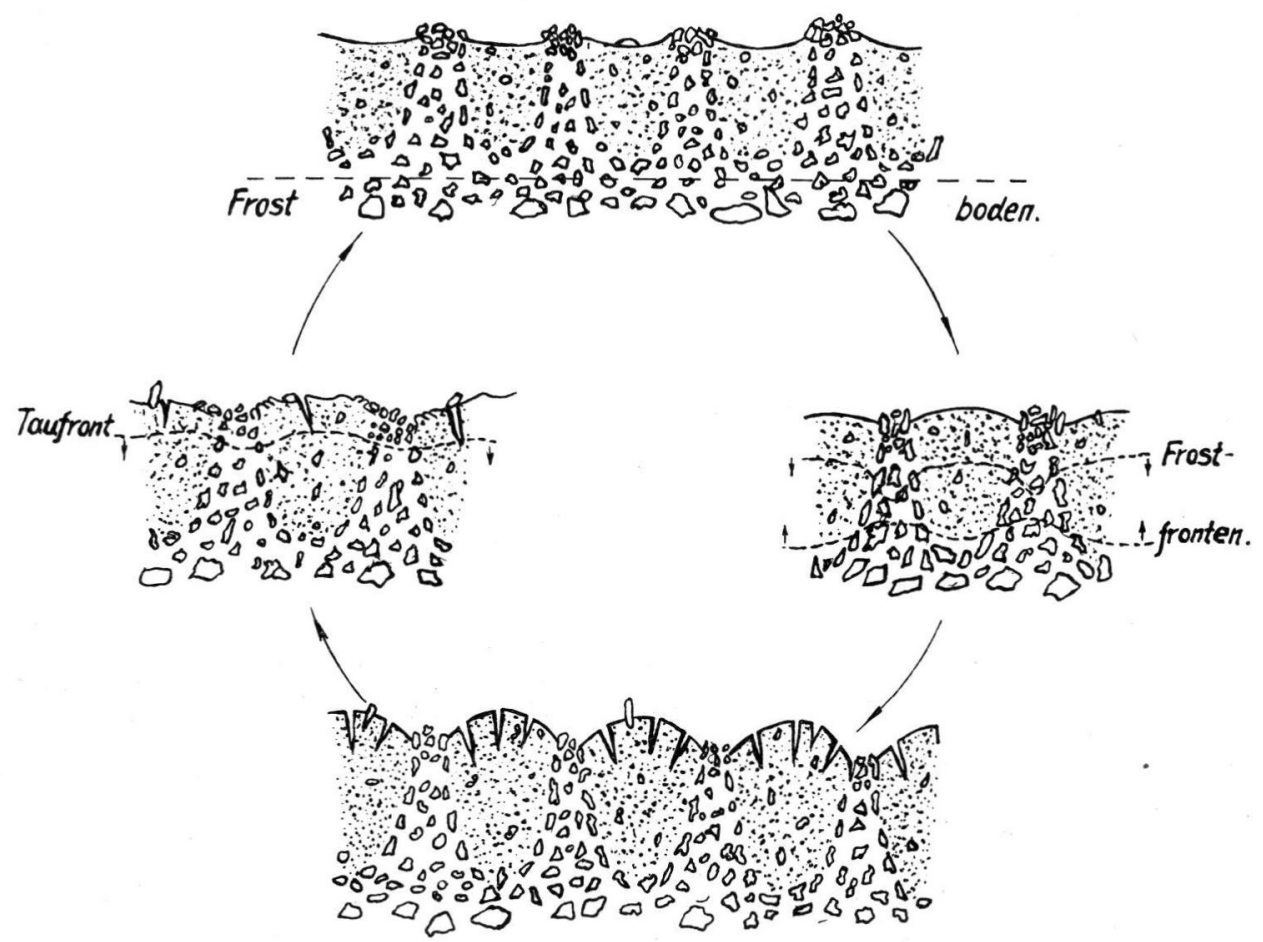

Abb. 1. Schematische Darstellung der Abläufe im Strukturboden (E. SchENK 1955). Der sommerlich oder täglich aufgetaute Boden wölbt und hebt sich unter der Einwirkung des Frostes, sogar bis zur Bildung von Rissen. Durch das Auftauen sinkt die so geschaffene Form wieder zusammen. Ihre Oberfläche liegt dann eingesenkt in den Pflanzen- oder Steinringen, -wällen und -netzen. Die bei dem Aufwölben mitgehobenen Steine oder Pflanzenpolster frieren allmählich aus und gleiten auf der aufgetauten breiigen Oberfläche des im übrigen noch gefrorenen Erdkerns zu den Rändern $a b$ und bilden hier die Ringe und Netze. 
standsformen ausgehen, sind sie von vornherein gegenstandslos. Hätte jedoch damals schon völlige Klarheit bestanden über die Beziehung zwischen Frost- und Auftauperiode einerseits und den Zustandsformen der Strukturen andererseits, so wären konsequenter Weise diese Theorien auch in den richtigen Weg eingelenkt worden.

\section{Zustandsformen und Bewegungsvorgänge}

Wenn die Feststellung, daß der $\mathrm{H}$ ü ge $\mathrm{l}$ ein Werk und eine Form des Winters, d. h. des Frostes ist und daß die im Steinkranz oder Pflanzenring eingesenkte Struktur mit ihrer breiigen Füllung nur eine Erscheinungs- und Zustandsform des Sommers, bzw. der A uftauperiode ist (Abb. 1), als gesicherte Erkenntnis angesehen werden darf, so klären sich die Widersprüche mit der Konvektionstheorie von selbst. Wie bereits vermerkt, sind die in Sommer aufgewölbten oder flachen Formen (Abb. 2) als inaktiv, als fossil oder quasifossil anzusehen. Die Bewegungsvorgänge, die zur Materialsortierung führen, sind an die feste gefrorene Form mit a uftauender Oberfläche gebunden und damit a uf die kurze Zeit des Auftauens beschränkt. Sie lassen sich im Schema einfach und klar darstellen (Abb. 1) als Folge der Solifluktion im kleinen Bereich, als Mikrosolifluktion, wie sie H. Posen (1934) und Th. Sörensen (1935) zuerst beschrieben haben.

Den Begriff der Solifluktion nun auf alle bewegenden Vorgänge der Frostwirkung zu erweitern, hieße aber, neue Unklarheiten und Schwierigkeiten schaffen, wie Verf. gezeigt hat (E. SCHENK 1954). Notwendigerweise müssen wir bei der ursprünglichen Vorstellung der Solifluktion bleiben (J. G. Andersson 1906), die von der Beobachtung des fließenden und sich bewegenden Bodens ausging und dabei mehr oder weniger bewußt an die Wasserübersättigung infolge des Frostes dachte. Ob dies als eine Folge der Tjäle (d. h. des Permafrostbodens) oder als unmittelbare Folge des Gefrierens angesehen wurde, ist unwesentlich. In der vom Verfasser gegebenen Definition der Soli-

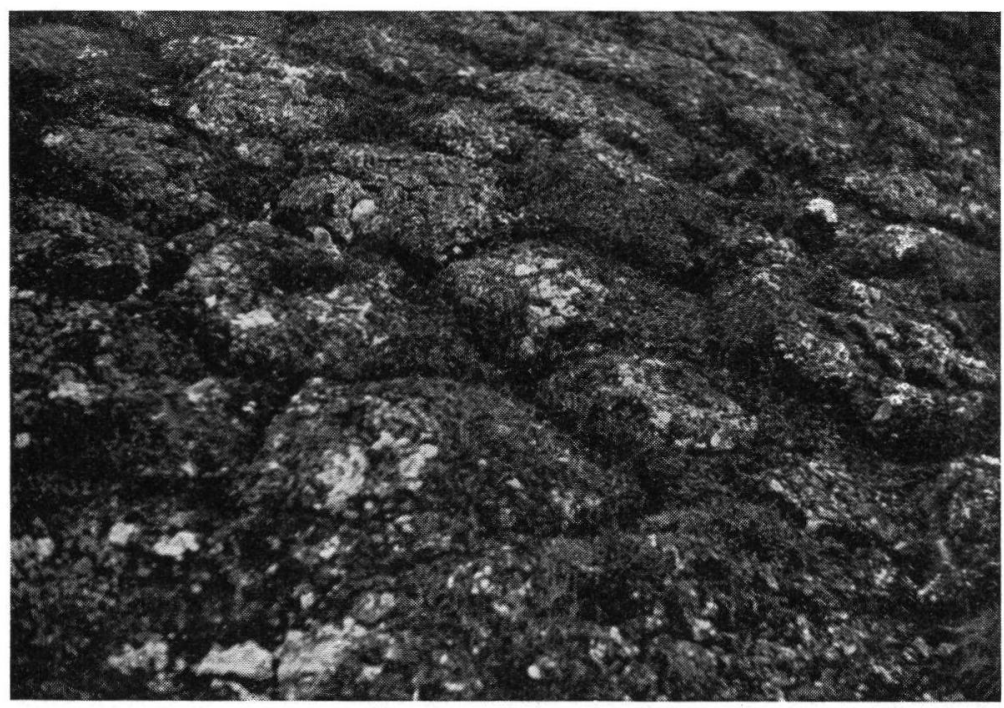

Abb. 2. Alte, fast fossile Polygone im lehmigen Feinschutt mit Moos- und Flechtenvegetation an der Liefdebay, Spitzbergen. Die Risse und Wölbungen bleiben auch im Sommer erhalten. Die geringe Aktivität dieser Strukturen ist auf die zentralen Teile beschränkt und hier kenntlich an der zerstörten Vegetationsdecke. Die einzelnen Strukturen sind jetzt der Gesamtgleitung der obersten Bodendecke $(\mathrm{ca} .30 \mathrm{~cm})$ eingeschaltet, wobei die einzelnen Zellen vielfach gekippt und gedreht werden. Aufn. v. Verf. 1936. 
fluktion (E. SCHENK 1954) dürfte ihr Wesen vollkommen erfaßt sein. S i e is t $\mathrm{n}$ ämlich definiert als die von der Gravitation bestimmte Fließbewegung des Bodens, derinfolge der Wasseranreicherung durch den Gefriervorgang beim Auftauen mit Wasser übersättigt wird.

Der von A. Hamberg (1915), so einfach und klar dargestellte Vorgang des Aufund Ausfrierens der Steine ist nun im Zusammenhang mit der Solifluktion eine erste Vorbedingung für die Aussortierung von Steinen und ihre Anreicherung an den Strukturrändern. Indem die Steine und dgl. nicht durch Frostschub, sondern durch die Gravitation auf der aufgetauten, schmierigen Oberfläche der gewölbten Strukturen abgleiten und mit dem fließenden Boden zum Rande transportiert werden, erfolgt mit jedem Ausfrier- und Auftauvorgang eine allmähliche Anreicherung der Steine an den Strukturrändern. (Abb. 3). Dasselbe gilt auch für die Pflanzen, die in den Zentren aktiver Strukturen stets fehlen! (s. a. Abb. 2). M. Hopkins (1951) hat.jüngst dieses Verhalten der Pflanzenpolster schön beschrieben. Durch eigene Fotos kann ich diese Vorgänge belegen. An frischen aktiven Formen wird der aufmerksame Beobachter immer Gleitspuren oder Raffungen in den Pflanzenringen beobachten können, eben jene Erscheinungen, die zur Vorstellung des Brodelns führen. Aber in voll aufgetauten Formen stehen sie im Widerspruch sowohl zu einer vom Zentrum nach den Rändern hin kreisenden Bewegung als auch zur zentral eingesenkten Oberfläche des Strukturkessels.

Was die Quellungs- und Konvektionstheorie als richtig und entscheidend ansieht und worüber im übrigen noch keine einmütige Auffassung bestand, war die Bedeutung des Wassers in quantitativer und qualitativer Hinsicht (s. a. C. Troll 1944), obwohl das Wasser von allen, die sich mit Froststrukturen befaßt haben, als wesentlich angesehen oder sein Vorhandensein im Untergrund als selbstverständlich angenommen wurde. Damit kommen wir nun zu den entscheidenden Problemen.

\section{Der Frostboden als kolloidales System}

An anderer Stelle habe ich zu zeigen versucht (E. Schenk 1955), daß in den Periglazialgebieten nur das W as ser für die Strukturentwicklung von ents cheidender Bedeutung ist, das zu Beginnder Frostperiode zurVerfügung

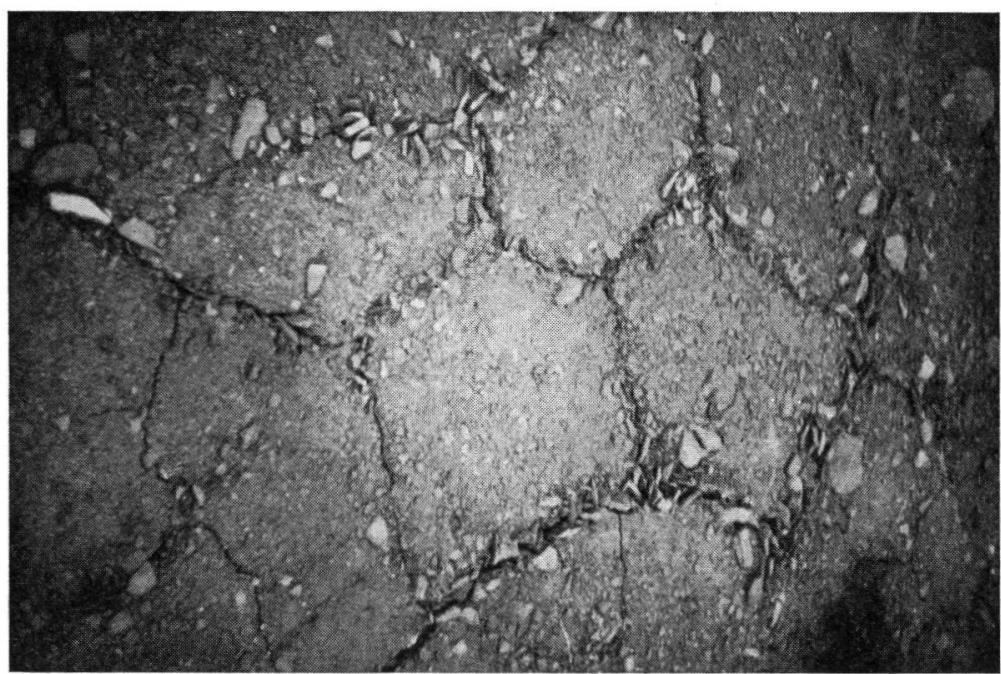

Abb. 3. Junge, sich noch entwickelnde Steinnetzpolygone mit Spalten, die durch Dehydratation entstanden und als solche sowohl beim Gefrieren als auch beim "normalen“ Austrocknen in Funktion treten. Liefdebay, Spitzbergen. Aufn. v. Verf. 1936. 
s te ht. Grundlegend sind ferner die experimentell gewonnenen Ergebnisse seit Jung (1931 u. 1933), G. Given (1925), S. Johannson (1914), St. M. TAber (1929 u. 1930), G. Beskow (1930), A. Dücker $(1939,1940,1942)$ und A. Casagrande (1934), wodurch die funktionalen Beziehungen des Wassers zu den Korngrößen im Frostboden aufgedeckt worden sind.

Die in den Frostgefährlichkeitskurven zum Ausdruck gebrachte Gesetzmäßigkeit ist im Grunde nichts anderes als die Auswirkung der hydratisierenden Teilchen des Mineralbodens von der Größenordnung 2:10-3 mm, d. h. der tonigen bzw. kolloidalen Teilchen, sofern a us reichende Wassermengen für Eisbildung zur Verfügung stehen. Die Eigenschaften der tonigen Bestandteile in Verbindung mit dem Wasser, also die Kolloidnatur des Bodens, entscheidet über Grad und Maß der Auswirkung des Frostes, m. a. Worten über die Strukturbildung. Erst von hier a us is t a u ch die Mannigfaltigkeit der Erscheinungsformen, und zwar sowohl die Variationsbreite der einzelnen Typen, als a uch die Vielzahl der Typengenetisch erfaßbar und eine genetische Einteilung überhaupt sinnvoll und möglich.

Die moderne Bodenkunde betrachtet den Boden, wie bereits gesagt, als ein dreiphasiges kolloidal-disperses System von Mineralteilchen, Wasser und Gas (Luft und Wasserdampf), und P. VAGELER (1932) hat dargelegt, daß in diesen Systemen dieselben einfachen elektrischen Kraftgesetze gelten wie in chemischen Lösungen und in aller Materie überhaupt:

1. Entgegengesetzt geladene Massenteilchen oder Pole ziehen sich an, gleiche stoßen sich $\mathrm{ab}$.

2. Die Kraft der Anziehung und Abstoßung ist umgekehrt proportional dem Quadrat der Entfernung (Coulomb).

Nach dem erstgenannten Gesetz vollzieht sich die Anlagerung von Gas- und Wasserteilchen an die Teilchen der festen Phase, d. h. der ein elektrisches Potential besitzenden Mineralkörnchen des Bodens und des Eises, also die Hydratation. Das 2. Gesetz bietet uns die Möglichkeit, genau festzustellen, wie groß die Kräfte der Anziehung nach Zahl und Stärke sind und auch welche Widerstände den sich bewegenden Teilchen entgegentreten können und wann ein Ausgleich der Ladung zwischen den Ionen und Dipolen der Mineralteilchen nach Größe und Zahl erreicht ist. Indem das Ausmaß der Hydratation abhängig ist von der Ladungskonzentration der Ionen, d. h. ihrem Potential (P), das durch das Verhältnis von Elektrizitätsmenge (e) zur Kapazität (c) bestimmt ist $\left(\frac{\mathbf{e}}{\mathbf{c}}\right)$, wird die Diëlektrizitätskonstante (D) der Medien zum bestimmenden Faktor; denn die Kapazität ist gleich dem Produkt von Diëlektrizitätskonstante und Radius des Teilchens bzw. des Ions $\left(P=\frac{\mathbf{e}}{\mathbf{D} \cdot \mathbf{r}}\right)$. Die Diëlektrizitätskonstante des Wassers ist rund 81, die des Eises rund 2 (abhängig von der Temperatur; in der Nähe des Schmelzpunktes des Eises sind die Dipole nicht sämtlich fest in das Kristallgitter eingefügt (J. N. Hummel 1943).

Daraus ergibt sich nicht nur die Hydratationsreihe der Ionen $(\mathrm{Li}>\mathrm{Na}>\mathrm{K}>\mathrm{Rb}>\mathrm{Cs}$; $\mathrm{Mg}>\mathrm{Ca}$; Abnahme der Hydratation mit wachsendem Ionenradius), sondern auch, was entscheidend ist, das viel höhere Potential des Eises ( $\left.\frac{e}{2 r}\right)$ gegenüber dem des Wassers ( $\left.\frac{e}{81 r}\right)$. Hierin liegt die Erklärung für die Was seranreicherung und Eisschichtenbildung im Frostboden. Dieses hohe Potentialder Eiskristalle, ihre Hydratationsenergie, gibt den Impuls und ist der Antriebsmotor für alle Strukturbildung. Es ist die Kraftquelle aller Bewegungen in dem gefrie- 
renden System der Suspension der Strukturkessel. Schließlich gibt sie auch den Impuls für die örtliche Entstehung und Entwicklung einer Struktur in einem inhomogenen Boden überhaupt.

Da nun die Dipolmomente der Wasserdampfteilchen um ein mehrfaches größer sind als die des flüssigen Wassers (P. VAgeler 1932, S. 30), so hat also auch der Wasserdampf eine bedeutsame Rolle in dem polydispersen System des Frostbodens. Die Verdichtung des Wasserdampfes zu Wasser bei der Anlagerung an feste Teilchen kennen wir als die Hygroskopizität und Benetzung der Stoffe. Man mag diesen Vorgang, der mit einer Wärmeentwicklung durch den Übergang von potentieller in kinetische Energie verbunden ist und daher die Gefriertemperatur herabsetzt, als einen ersten ansehen bei der Bildung der Schwarmwasserhüllen um die Partikel des Bodens, denn die Anlagerung flüssigen Wassers folgt dann schnell nach. Jedenfalls müssen wir diesem Vorgang die entscheidende Bedeutung für die ungeheure Tiefenentwicklung des Dauerfrostbodens und speziell für die sogenannten Eiskeilbildungen, der Entwicklung mächtiger vertikaler Eisgänge, und bei der Eisschichtenbildung überhaupt zumessen. Sie entstehen bei genügender Frosteinwirkungszeit auch dort, wo der Wassernachschub aus einer Reserve nicht mehr möglich ist, sondern auf die Menisken und das Porenwinkelwasser und schließlich sogar auf die inneren Wasserhüllen der Mineralpartikel über die Bildung von Wasserdampf zurückgreifen muß. D i es k a n nur erfolgen durch die bedeutenden Energiedifferenzen zwischenden hydratisierten Gesteins-und Bodenpartikelchen und den hydratisierenden Eiskristallen, die einem dynamischen A usgleich, einem Gleichgewicht, zustreben. Im Frostboden haben wir beim Gefrieren und Auftauen also keine statischen Gleichgewichte, sondern immer nur vorübergehende dynamische Zustände vor uns.

\section{Hydratation}

Diese Energiedifferenzen sind osmotischer Natur, und ihr Wesen besteht in der Absättigung der elektrischen Feldkräfte der Korpuskeln durch Anlagerung von Wassermolekülen, also in der Hydratation (P. VAGELER 1939, S. 104). Die Wasserteilchen werden wie in einer Lösung umso energischer festgehalten, d. h. absorbiert, je weniger auf die einzelnen Korpuskeln kommen, d. h. je konzentrierter die Lösung ist. Dieser osmotische Druck ist meßbar und berechenbar aus der Korpuskularnormalität und dem Normaldruck. Die Rechnungen der Bodenkundler haben ergeben, daß mit Drucken von 50 at und viel mehr die Wasserhüllen von den Bodenteilchen festgehalten werden und die Dicke der Wasserhülle rund $1 / 4$ des Teilchenradius ist. Wir verstehen damit, daß auch der Quellungsdruck osmotischer Art ist. Die Grenze ist diesem gesetzt, auch bei großem Wasserüberschuß, durch den Schichtlastdruck, der mit der Tiefe der Struktur schnell zunimmt, wie sich auch aus den Quellungsversuchen eindeutig ergibt ( $\mathrm{H}$. GREeN 1928). Wir können durch Quellung also überhaupt keine tiefen Sitrukturen erklären.

Bedeutsam aber sind die Vorgänge der Quellung in dem Froststrukturboden in anderer Hinsicht. Sobald eine stauende Schicht im Boden vorhanden ist, reichert sich über ihr Wasser an. Demzufolge bekommen die Bodenteilchen in diesem Wasserhorizont einen Auftriebseffekt. Je näher sie der Oberfläche liegen, desto besser kann er sich auswirken. Die Folge ist eine Verringerung der Widerstände, die einer Hydratation der Bodenteilchen durch Schichtlastdruck, Packung usw. entgegenstehen und damit einer vollen Hydratation der Korpuskeln (= maximale Wassermenge = minimale Wasserkapazität des Systems). Sie beträgt das 4-5fache des Wertes der Hygroskopizität (P. VAGELER 1932, S. 109). Dieser Umstand ist es, der dem lehmigen 
Kessel der aktiven Strukturen den Charakter der Suspension verleiht. Nach der Tiefe hin ändert sich dieser Zustand schnell, der Kesselinhalt wird fester.

Der Auftriebseffekt kann andererseits nicht in Anspruch genommen werden für die Materialtransporte im Kessel und die Aussortierung grober Korngrößen, ihre Anreicherung an den Rändern der Strukturen ${ }^{1}$ ). Er wirkt flächenhaft. Die groben Partikel werden infolge ihrer kleinen Oberflächen und ihres geringen Hydratationsvermögens und der stärkeren Gravitation eher sinken als steigen, geschweige denn eine kreisende Bewegung vollziehen, die für eine homogene Lösung bzw. eine Suspension mit ganz einheitlich großen Kolloidteilchen als Folge von Dichteunterschieden denkbar wäre. In solchen aktiven wasserreichen Strukturen aber liegen die wärmsten und dabei wegen ihrer größeren Hydratation spezifisch leichtesten Schichten stets oben, bzw. oberflächennahe. Sie bilden eine lockere Schlammschicht, die nach der Tiefe hin immer fester, d. h. dichter gepackt ist und damit auch ein größeres spezifisches Gewicht hat als die oberen Schichten. Ubberdies hat sie eine verhältnismäßig so geringe Mächtigkeit, daß Konvektionsbewegungen niemals in große Tiefe strukturbildend reichen können.

\section{Dehydratation und Eiskeilbildung}

Für die Strukturbildung erheblich wichtiger als die Quellung selbst ist der ihr entgegengesetzte Vorgang: die Schrumpfung. Sie entspricht dem Wasserentzug bis zu dem Augenblick, wo die Hydrathüllen so verringert worden sind, daß die Korpuskeln sich gegenseitig berühren und damit das sogenannte Minimalvolumen (P. VAGELER 1932, S. 109) einnehmen. Aus dem verbleibenden $Z$ wischenraum der Poren kann das Wasser nur als Dampf entzogen werden. Die dabei nunmehr auftretende Volumenverringerung, die sogenannte Restschrumpfung, ist kleiner als dem Wasserentzug entspricht. Experimentell haben St. M. TABER (1930) und A. DüCKER (1940) diese Schrumpfung in Frostböden gezeigt. Da die Schrumpfung im naturlichen Boden die unvermeidliche Folgedes Wasserentzuges ist, erscheint sie als die einzige Möglichkeit, die wabige Strukturdes Frostbodens und sein Wachsen in die Tiefe $z u$ erklären. Darüber hinaus macht sie auch die Eiskeilbildung verständlich, denn Kontraktionsrisse infolge tiefer Temperaturen können, wie St. M. TABER (1949) schon dargelegt hat, dafür nicht in Frage kommen. Diese ArtKontraktion ist rückgängiggemacht, ehe ein Tropfen Wasserzur Auffüllung der Spaltenzur Verfügung s te h t. Anders ist das bei Schrumpfungsrissen. Hier können die Eiskristalle von den horizontalen Eislagen aus in die Spalten hineinwachsen und Wasserteilchen auch noch in Dampfform aus dem Boden anziehen, anlagern und in ihr Gitter einbauen. Sobald die Eisbildungen tiefer reichen als der sommerliche Auftauboden, bleiben sie erhalten, und stets wirkt dann ihre Hydratationsenergie. Bei dem Wiedergefrieren des Auftaubodens wirkt sie auch von unten herauf, wie aus der Temperaturkurve im Dauerfrostboden klar hervorgeht (Abb. 4). In dem einmal geschaffenen Schrumpfungsriß wird Wasser als feinster Film festgehalten, und es ist eine seit S. JoHAnnson bekannte Tatsache, daß die Eisblätter und - gänge immer wieder an derselben Stelle entstehen, auch im Experiment (A. Dücker). Ebenso ließen sich polygonale Risse und Spaltenmuster experimentell erzeugen (A. Dücker, St. M. TABER). In der Natur beobachten wir sie an offenbar ganz jungen, entstehenden Strukturen (Abb. 3) als randliche Begrenzung mit und ohne Steinnetzmuster. Sie sind Folgen

1) Die von K. Gripp (1952) geschilderten Experimente sprechen nicht dagegen. Eine physikalische Beschreibung ist dabei leider nicht gegeben worden. Soweit die mitgeteilten Versuchsbedingungen überhaupt eine Beurteilung zulassen, waren die beobachteten Bewegungen durch die Luft, die den Bodenteilchen bei Beginn der Versuche angelagert war, verursacht. Sie wirkte als Benetzungswiderstand, der in einem physikalisch definierbaren kritischen Punkt von der Hydratationsenergie überwunden wurde und die Iufthüllen der Teilchen damit freigab. 


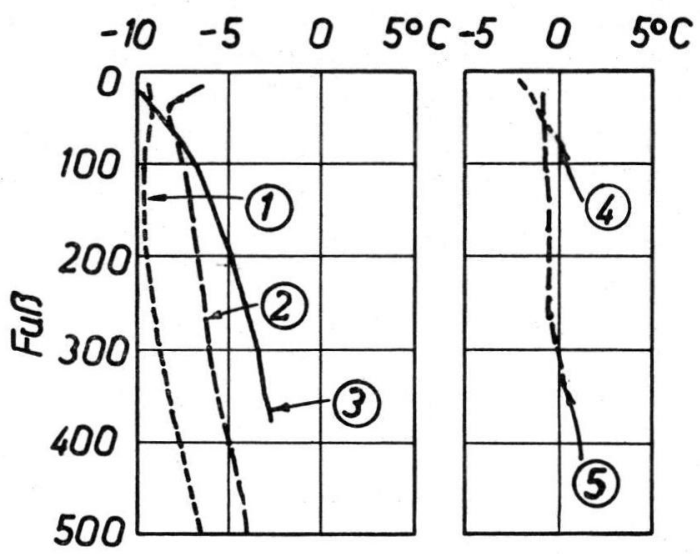

Abb. 4. Temperaturverhältnisse im Dauerfrostboden a) im zusammenhängenden Permafrostgebiet, b) in Permafrostrestgebieten (Dauerfrostinseln). (1) in einer Bohrung 25 Meilen südwestlich von Barrow, Nord-Alaska, (2) in einer Bohrung in Barrow, Nord-Alaska, (3) in einer Bohrung bei Yakutsk, UdSSR, (4) in einer Bohrung bei Nome, Alaska, (5) in einer Bohrung bei Bukachacha, UdSSR. Aus Robert F. Black: Permafrost, in Trask's Applied Sedimentation, 1950.

des Wasserentzuges, der Dehydratation der Bodenteilchen. In ihnen kann sich das Wasser wieder ansammeln vor Beginn des Gefrierprozesses, und zwar schneller als die Quellung folgen kann. Aber auch der Gefriervorgang kann von hier aus angreifen und noch Wasser für die Eisbildung aus dem Boden herausziehen. Jedenfalls zeichnet der Schrumpfungsriß den vertikalen Eisbildungen immer wieder Ausdehnung und Wachstum vor. Dort, wo diese dann die Auftauperiode überdauern und mehr oder weniger nur der Kessel der Struktur ganz auftaut, wachsen sie bei jeder neuen Frostperiode in die Breite. Ihre Erhaltung während der Auftauzeit wird begünstigt oder vielleicht sogar erst möglich durch die Überdeckung mit dem Schutt, der von der auftauenden Frostform zu den Rändern abfließt, oder durch Pflanzenpolster. Die Schuttanreicherungaufden Eisgängen zwischenden Strukı urengibtspäter wiederum Anlaßzuneuen Strukturbildungen. Während der Auftauzeit erscheinen sie als schlammerfüllte Gräben und Rinnen in den Eiswällen, die die Strukturen umgrenzen.

Bei der Deutung und Erklärung dieser Großformen ist die Zeitdauer zu berücksichtigen. Ihr Breiten- und Tiefenwachstum erfolgt mit dem Vorrüicken der Dauerfrostböden in den Periglazialgebieten, also seit Beginn der Eiszeit. Nach diesen Überlegungen haben wir in ihnen also sicherlich schon sehr alte Formen vor uns, ebenso wie im Dauerfrostboden überhaupt. (St. T'Aber 1949, J. BüDEL 1954)

Horizontale Eisschichten und vertikale Wasserbewegung

So wie die vertikalen Eisblätter als Folge der Hydratation der Eiskristalle anzusehen sind, so sind auch die horizontalen Eislagen ein klarer Ausdruck und ein Abbild der osmotischen Druckdifferenzen im gefrierenden Boden. Der Dauerfrostboden erscheint gneisartig gebändert (St. M. TABER 1930; Abb. 5). Auch im Experiment (St. M. TABER 1930, A. DüCKer 1940) entsteht diese Struktur. Dabei fällt die Rhythmik im Wechsel und in der Mächtigkeit der Boden- und Eisschichten auf. Sie ist umso ausgeprägter, je gleichmäßiger und je gleichkörniger der Boden ist.

Die Erklärung für diese Erscheinungen geben uns die Gesetze der W a s s e r b e w e g u $\mathrm{ng}$ im Boden, die $\mathrm{K}$ in etik des Wassers in einem polydispersen System. In fast 
allen bisherigen Erklärungen der Mechanik und Dynamik der Frostbodenstrukturen steht die Kapillarität des Bodens im Vordergrund. Sie wird als Hauptmittel der Wasserbewegung und kapillarer Unterdruck als ihr treibendes Moment angesehen. Auf ihre Beziehungen zu den Korngrößen gründet man schließlich auch die Kriterien der Frostgefährlichkeit. Nach den Untersuchungen der modernen Bodenkunde $\mathrm{k}$ a n n de r Ka pillarität aber nur eine sehr untergeordnete, unwesentliche Bedeutung zugemessen werden, sofern ihr überhaupt eine zu$\mathrm{k}$ o $\mathrm{mmt}$.

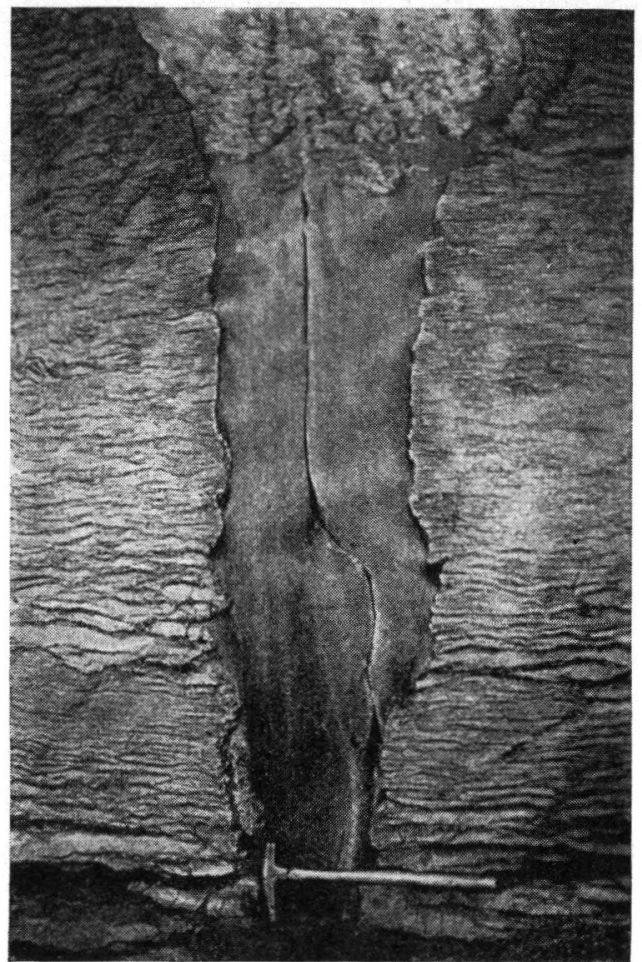

Abb. 5. Rhythmische Eisschichtung und Eiskeilbildung im Dauerfrostboden von Nord Alaska. Aus St. M. TABER 1930.

Was als kapillare Steighöhe bezeichnet wird, ist die Länge der getragenen, an den Enden einen Meniskus bildenden Wassersäule in Kapillarröhrchen ïber dem Grundwasserspiegel. In vollkommen benetzten Haarröhrchen ergibt sich die bekannte Beziehung von Oberflächenspannung des Wassers zum Durchmesser der Röhrchen $(\mathrm{H}=$ $\frac{14,82}{\mathrm{r}} \mathrm{mm}$; bei $20^{\circ} \mathrm{C}$ ). Danach ist also die kapillare Steighöhe leicht zu berechnen, und es ergibt sich dann, daß Tone, wie auch fast noch immer angegeben wird, eine sehr hohe kapillare Steighöhe hätten. Diese theoretischen Ergebnisse stehen aber im Widerspruch zu den tatsächlichen Verhältnissen, denn in Tonen beobachten wir praktisch k e in e Kapillarität oder höchstens eine sehr kleine. P. VAGELER (1933, S. 115) hat auch bereits vor mehr als zwanzig Jahren im einzelnen gezeigt, daß von kapillarer Hebung oder kapillarer Bewegung des Wassers im Boden fast gar keine Rede sein kann und daß die Kapillaren des Physikers nichts mit den Porenräumen der Bodenkundler zu tun haben. Er hat ferner gezeigt, daß ein kapillarer Hub nur dann auftritt, wenn die Schwarmionen der Bodenkorpuskeln sich hydratisieren, d. h. wenn die Moleküle der Flüssigkeit von den Phasengrenzflächen (der Bodensubstanz mit ihrer 
Kationenbelegung) sorbiert werden, also wenn elektrische Kraftfelder zwischen fester Substanz und Flüssigkeit sich ausgleichen. Eine kapillare Depression tritt dann auf, wenn gleichsinnige Kraftfelder sich abstoßen. Nach P. VAgELer sind also die positiven und negativen elektrischen Feldkräfte das be wegende primäre Moment, das auf die Flüssigkeit wirkt. Die Menisken, ihr Auftreten und ihre Form, sind nur se-kundärer Art und bestimmt von den elektrischen Kräften, die auch die Benetzbarkeit und Unbenetzbarkeit bedingen. Sogenannte Verunreinigungen sättigen z. B. die elektrischen Feldkräfte $a b$ und heben damit den Widerstand gegen die Benetzung auf. An trockene Mineralpartikel angelagerte Luft - die übrigens immer leicht im auftauenden Frostboden eingeschlossen wird ${ }^{2}$ ) - übt ebenfalls einen für flüssiges Wasser schwer überwindbaren Benetzungswiderstand aus. Leicht überwindbar dagegen ist er für Wasserdampf, da dessen Dipolmoment dreimal so groß ist als das des flüssigen Wassers (P. VAgeler S. 119). Ein Meniskus bildet sich erst bei Berührung der Wasserhüllen der einzelnen Bodenpartikelchen und des Wasserfilmes, der als kontinuierliche Hülle flüssigen Wassers von Teilchen zu Teilchen reicht, solange eine Dampfspannung vorhanden ist (funikulares Wasser im Gegensatz zu pendiculärem, wo das Wasser nesterweise in den Porengängen auftritt und eine Wasserbewegung in flüssiger Form nicht mehr möglich ist). Hängt nun die Steighöhe des sogenannten kapillaren Wassers ganz $a b$ von der Reibung, wie P. VAGELER darlegt, so gilt das erst recht für den Wassernachschub, den Wasserzufluß zur Gefrierfront. Dieses aufsteigende Wasser kann sich nicht gemeinhin durch die Porenräume bewegen, sondern es stehen ihm zunächst nur die Zwischenräume zwischen den Bodenpartikeln $\mathrm{m}$ it ihren absorbierten, festgehaltenen Wasserhüllen zur Verfügung: das sogenannte spannungsfreie Porenvolumen. Das sich hierin bewegende Wasser ist das wirkliche Kapillarwasser, dessen "Steighöhe" normalerweise (bei der Sickerung) abhängig ist von der Tragkraft des Meniskus. Je feiner die Bodenteilchen, desto bedeutungsloser wird es. Maßgebend sind bei dieser Wasserbewegung natürlich die engsten Porenquerschnitte.

Der durch die Saugkraft der Eiskristalle erzeugte Unterdruck im wassergesättigten Boden bewirkt schließlich eine auf das Hüllwasser beschränkte Wasserbewegung. Sie greift die minimale Wasserkapizität des Systems an, und zwar bis z u dem Punkt, wo sie durch die Widerstände, die Reibung und Hydratationsenergie der Teilchen kompensiert ist. Je feiner die Bodenpartikel wie in Tonen - desto größer sind diese Widerstände, und der Wassernachschub muß schließlich abreißen. Hier hört die Eisschichtenbildung auf, und es gefriert der Boden „kompakt", d. h. mit an Ort und Stelle verbliebenem Hüll- und Porenwinkelwasser. Der Augenblick merklicher Verringerung der Wassernachlieferung kennzeichnet den sogenannten lentokapillaren Punkt. Hier ungefähr beginnt die Wasserbewegung mittels der Dampfphase, soweit die hygroskopischen Kräfte dazu Spielraum lassen. Im Frostboden scheint somit der lentokạpillare Punkt durch die Grenzen der Eisschichten gekennzeichnet und sichtbar festgehalten $\mathrm{zu}$ sein.

Wir sehen daraus, daß die von P. VAGELER im Nichtfrostboden erkannten Vorgänge auch für die Mechanik und Dynamik der Strukturbildung im Frostboden entscheidend sind. Die Eisbildung und Bewegung des Wassers ist nur einganz anfänglich von vorhandenem Kapillarwasser, im übrigen aber ganz vom Hydratwassergenährter Vorgang, der ron den Hydratationsenergien der beteiligten Komponenten, d. h. dem os motischen Druckgefälle, gesteuert wird. Die von St. M. Taber und A. DüCKER vorgelegten Diagramme über die Frosteindringung, den Wassernachschub und die Frosthebung belegen dies überzeugend.

$\left.{ }^{2}\right)$ Ihre Existenz in den aufgetauten aktiven Strukturen ist ebenfalls ein Beweis gegen die Brodeltheorie. 


\section{Frosteindringung}

Die Frosteindringungsgeschwindigkeit nimmt merklich ab mit der Zunahme der feinen Korngrößen, d. h. mit der Zunahme des Hydratwassers und der Hydratationsenergie entsprechend der Entwicklung der Oberflächen, bzw. der Phasengrenzflächen der festen zur flüssigen Substanz (Abb. 6). Die Verringerung oder Bremsung, wie wir sagen können, ist umso auffälliger, je größer das Temperaturgefälle und damit die osmotische Saugkraft ist, die auf das von den Bodenteilchen freizumachende Hydratwasser wirkt. In den Sanden mit ihrer geringen Komplexbelegung und daher geringen

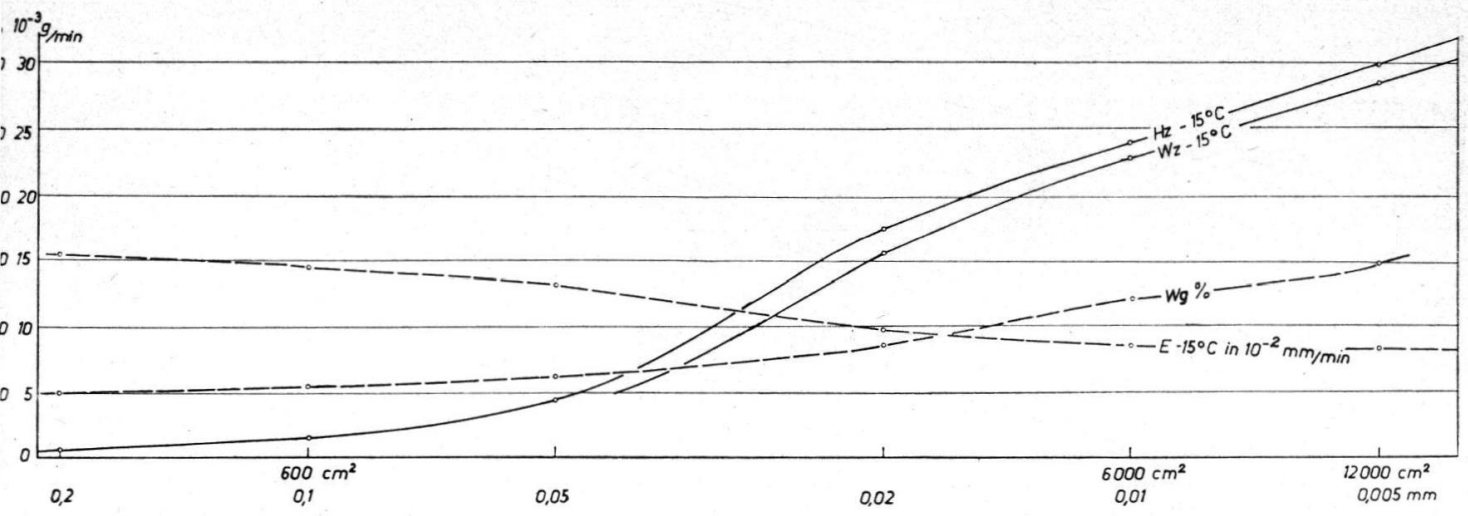

Abb. 6. Die Beziehung der Korngrößen (in $\mathrm{mm}$ ) und Kornoberflächen (in $\mathrm{cm}^{2}$ pro $\mathrm{cm}^{3}$ Boden) zur maximalen Wasseraufnahmefähigkeit (Wg in \% des Trockengewichtes, Enslinbestimmung) und zur tatsächlichen Wasseraufnahme $\left(\mathrm{W}_{\mathrm{Z}}\right.$ in $10^{-3} \mathrm{~g} / \mathrm{min}$ und \%), Frosthebung $\left(\mathrm{Hz}_{\mathrm{Z}}\right.$ in $10^{-3}$ $\mathrm{mm} / \mathrm{min}$ ) und Frosteindringungsgeschwindigkeit ( $\mathrm{E}$ in $10-2 \mathrm{~mm} / \mathrm{min}$ ) bei einer Frosteinwirkung von $-15^{\circ} \mathrm{C}$; dargestellt nach experimentellen Untersuchungen von A. DüCKER (1939) im einfach logarithmischen Maßstab. - Die Fähigkeit des Bodens, Wasser aufzunehmen, wächst mit der Vermehrung der Teilchenoberflächen und ist begrenzt. Der Erhöhung des Gehaltes an angelagertem Wasser entspricht die Verringerung der Frosteindringungsgeschwindigkeit, so daß sich beide Kurven überschneiden, und zwar im Bereich der Teilchengrößen um 0,02 mm, dem Bereich gefährlichster Frosthebung (s. Abb. 7). In diesem Bereich liegt auch der Wendepunkt der Kurve der Frosteindringungsgeschwindigkeit. Ihm zugeordnet ist der Wendepunkt in der Kurve der durch den Frost bedingten Wasseraufnahme. Diese nimmt von hier an ab und korrespondiert genau mit der Frosthebung.

Hydratationsenergie dringt der Frost am schnellsten vor, da hier keine großen osmotischen Druckgefälle entstehen können. Wir verstehen auch, daß bei Absättigung der Feldkräfte dieser gröberen Partikel durch die höheren Dipolmomente der Eiskristalle das Hydratwasser gewissermaßen abfällt und das Wasser von der Gefrierfront scheinbar fortgedrückt wird.

\section{Frosthebung}

Die Frosthebung ist nach den bekannten Diagrammen (St. M. TABER, A. Dücker) im wesentlichen porportional dem Wasserzufluß plus der Hebung durch das primär vorhandene Wasser. Wir erkennen jetzt, daß der Wasserzufluß aber wiederum proportional zu sein scheint der Zunahme der Oberflächen mit der Zunahme der Feinkörnigkeit, sobald diese eine Größe von $6000 \mathrm{~cm}^{2} / \mathrm{cm}^{3}$ (Schluff und Ton) erreicht haben, also bei einer Korngröße von $0,01 \mathrm{~mm}$ (Abb. 7). Dieses Bild ergibt sich, wenn man die Beziehungen zwischen Wasserzufluß und Korngröße nach den Untersuchungsergebnissen von A. DüCKER (1939 und 1940) umrechnet. Vermutlich läßt sich die Grenze der Frostgefährlichkeit nach diesen Gesichtspunkten experimentell schärfer fassen als bisher. Die Kurve zeigt einen steilen Anstieg im Bereich der Korngrößen, die größer sind als $0,01 \mathrm{~mm}$ und kleiner als $0,1 \mathrm{~mm}$, also bei den Oberflächen von $600-6000 \mathrm{~cm}^{2}$ / 
$\mathrm{cm}^{3}$ (Feinsand). Die Zuflußmenge, d. h. auch die Nachlieferungsgeschwindigkeit, steigert sich ebenfalls in einem offenbar konstanten Verhältnis zum Temperaturgefälle, wie die Diagramme von A. DüCKER und St. M. TABER zeigen, also wie wir jetzt sagen können, mit der osmotischen Druckdifferenz zwischen Frostfront (Eisfläche) und Hüllwasser.

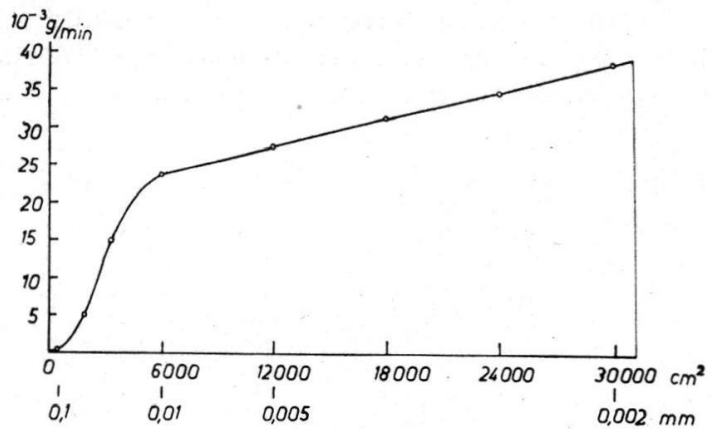

Abb. 7. Die Hydratation der Bodenpartikel in Abhängigkeit von der Teilchengröße und der Teilchenoberfläche bei Frosteinwirkung von $-15^{\circ}$ Celsius; dargestellt nach experimentellen $\mathrm{Er}$ gebnissen von A. DücKer (1939 c). Die Wasseraufnahme im gefrierenden Boden und damit die Hydratation erfolgt bei den gröberen Teilchen mit einer Oberfläche von weniger als $6000 \mathrm{~cm}^{2} / \mathrm{cm}^{3}$ außerordentlich schnell. Nach Überschreitung dieses Wertes verringert sich der Wasserzufluß allmählich mit der Zunahme der Durchflußwiderstände infolge der Vergrößerung der Oberflächen. Der Verlauf dieser Kurve entspricht nicht der theoretisch aus der Korngröße und dem zugehörigen Porenquerschnitt berechneten Kapillarität mit den außerordentlichen Steighöhen bei zunehmender Kornfeinheit, sondern der Wasseraufnahme infolge osmotischer Druckdifferenzen nach P. VAGELER.

Erklärbar erscheint dies durch das in diesem Korngrößenbereich noch mögliche Kapillarwasser. Bei der Einwirkung des osmotischen Unterdruckes kann sich hier noch das spannungsfreie Porenvolumen auswirken, das mit zunehmender Kornfeinheit immer geringer, während die Reibung dagegen immer größer wird. Im feinen Sand bzw. Schutt aber genügt bereits ein geringes osmotisches Gefälle zur Gefrierzone, um verhältnismäßig große Wassermengen in Bewegung zu bringen. Hier erfolgt also auch eine dementsprechende schnelle Frosthebung. Im groben Sand dagegen mit seinen kleinen Oberflächen und seiner geringen Komplexbelegung erfolgt keine Hebung und $\mathrm{Be}-$ wegung trotz seines großen Porenvolumens und seiner guten Durchlässigkeit. Dieses merkwürdige und bisher unerklärliche Verhalten ist durch die chemisch-physikalischen Eigenschaften der Bodenpartikel verständlich.

\section{Len tokapillarer Punkt}

Mit der Aufzehrung der Wasserreserve für die Nachlieferung zur Gefrierfront wird das Hydratwasser, wie bereits vermerkt, bei genügend starker Frosteinwirkung angegriffen. Damit wird die Dicke der Hydrathüllen verringert und das spannungsfreie Porenvolumen vermehrt, die Reibung also vermindert. Die Kurven des Wasserzuflusses in der Zeiteinheit müssen dementsprechend einen steilen Endabschnitt zeigen, der mehr oder weniger plötzlich in eine sehr flache Lage umbiegt, sobald der lentokapillare Punkt erreicht ist und die Dampfphase beginnt. Solche Steilanstiege sind offenbar auch vorhanden, wenn man sich die bekannten Diagramme daraufhin ansieht. Der abgerissene Wasserfaden kann erst wieder aufgenommen werden, wenn die Gefrierfront tiefer eingedrungen ist und damit das Hydratwasser wieder in den Wirkungsbereich der Absorption der Eiskristalle fällt. Damit beginnt eine neue Eisschichtenbildung. P. V $\mathrm{V}_{\mathrm{A}}$ GELER (1932, S. 130) spricht von os mot is chem Radius und kritischer Schichtdicke. Diese Größen dürften kaum besser erfaßbar sein als durch das Gefrierexperi- 
ment, das sie im rhythmischen Wechsel von Eis- und Bodenschicht unmittelbar zum Ausdruck bringt. Dieser osmotische Radius dürfte auch das Geheimnis der Dimensionierung der Strukturen sein.

\section{Rückblick}

Rückschauend müssen wir festhalten, daß die moderne Bodenkunde die Probleme des Frostbodens in neuem Licht $\mathrm{zu}$ betrachten und $\mathrm{zu}$ untersuchen ermöglicht. Die bisher durchgeführten Experimente von Straßenbauern und Bodenmechanikern erscheinen dabei noch nicht erschöpfend und es gilt, sie unter diesen neuen Gesichtspunkten durchzuführen und die bisherigen Ergebnisse zu verbessern und zu präzisieren. Umgekehrt dürfte das Gefrierexperiment die Möglichkeiten der kolloidchemischen Untersuchung des Bodens erweitern und die Fragen der Kapillarität und Hydratation, die Untersuchung der Oberflächen und Grenzflächen, der verschiedenartigen Beiwerte, der Durchlässigkeit usw. und anderer spezifischen Eigenschaften des Bodens klären und erforschen helfen.

\section{$\mathrm{Zus}$ a m m en a s sung}

Zusammenfassend ist zu sagen, daß die Erscheinung des Dauerfrostbodens, die Bildung der Eiskristalle und Eisschichten in horizontaler und vertikaler Lage, die polygonalen Netze und Strukturen, die Hebung und Solifluktion und ihre Folgen durch nichts besser erklärt werden können, als durch die Vorgänge und Gesetze der Hydratation und Dehydratation. Diese Abläufe im Froststrukturboden werden bestimmt und gesteuert von dem osmotischen Druckgefälle, das durch die hohen Dipolmomente der Eiskristalle erzeugt wird. Möglich aber war eine solche Erklärung erst, wie die Geschichte der Strukturbodenforschung lehrt, nachdem Klarheit geschaffen war über die tatsächliche Mechanik der Strukturen, wozu wiederum die Erkenntnis von aktiven und inaktiven Formen im rezenten Formenschatz die erste Voraussetzung war.

\section{Literaturverzeichnis}

Alten, F. \& Kurmies, B.: Die physikalische Beschaffenheit des Bodens. - Handb. d. Bodenlehre; Ergänzungsb. Berlin 1939.

Andersson, J. G.: Solifluktion, a component of subaerial denudation - Journal of Geol. 14, 1906.

Beskow, G.: Erdfließen und Strukturböden der Hochgebirge im Lichte der Frosthebung. - Geol. Fören. i Stockholm Förhandlg. 52, 1925.

BRunt, H.: Structure earth. - Nature 140, 1925.

BüDEL, J.: Die "periglazialen" morphologischen Wirkungen des Eiszeitklimas auf der ganzen Erde. - Erdkunde \%, 1953.

Casagrande, A.: Bodenuntersuchungen im Dienst des neuzeitlichen Straßenbaues. - Der Straßenbau 25, 1934.

DüCKer, A.: Neue Erkenntnis auf dem Gebiet der Frostforschung. - Die Straße 17, Berlin 1939 (a). - - Der Einfluß von Salzlösungen auf das Gefrieren von Böden. - Ebendort 1939 (b). - - Untersuchungen über die frostgefährlichen Eigenschaften nichtbindiger Böden. - Forschungsarbeiten aus dem Straßenwesen Bd. 17, Berlin 1939 (c). - - Frosteinwirkung auf bindige Böden. - Straßenbau-Jhrb. 1939/40, Berlin 1940. - Uber „Bodenkolloide“ und ihr Verhalten bei Frost. - Der Bauingenieur, Zeitschr. f. d. ges. Bauwesen 23, 1942.

Fujrwhara, S.: Notiz über Strukturboden am Mt.Norikura (Zentraljapan.) - Tirigakuhyôron 4, 1928.

Given, G.: Kolloidale Eigenschaften des Tones und ihre Beeinflussung durch Kalksalze. - Diss. Göttingen 1915 .

Green, H.: Soil permeability in the Eastern Gezira. - J. agricult. Sci. 18 III, 1928.

GrIPP, K.: Uber Frost- und Strukturböden in Spitzbergen. - Ztschr. d. Ges. f. Erdk., Berlin 1926. - - Beiträge zur Geologie von Spitzbergen. - Abh. naturw. Ver. Hamburg 21, 1927. - - Glaziologische und geologische Ergebnisse der Hamburgischen Spitzbergen-Expedition 1927. - Ebendort 22, 1929. - - Gletscher und Bodenfrost, rezent u. diluvial. Geol. Rundsch. 21, 1930. - - Zwei Beiträge zur Frage der periglazialen Vorgänge. Meyniana, Veröff. geol. Inst. d. Univ. Kiel 2, 1952. 
Gripr, K. \& W. Simon: Experimente zum Brodelbodenproblem. - Cbl. f. Min. usw. Jg. 1933, Abt. B. - - Die experimentelle Darstellung des Brodelbodens. - Die Naturwiss. 22, 1934. - - Nochmals zum Problem des Brodelbodens. - Cbl. f. Min. usw. 1934.

HamberG, A.: Zur Kenntnis der Vorgänge im Erdboden beim Gefrieren und Auftauen. - Geol. Fören. Förhandlg. 35, 1915.

Нӧявом, В.: Einige Illustrationen zu den geologischen Wirkungen des Frostes auf Spitzbergen. - Bull. Geol. Inst. Upsala 9, 1908/09.

Hopkins, M. \& Sigafoos, R. R.: Frost Action and Vegetation Pattern on Seward Peninsula Alsaka. - Geol. Survey Bull. 974 - C., Washington 1951.

Hudino, Y.: Thermal Convektion of Liquids laden with some Powder. - Report of the Aeronautical Research Institute Tôkyô Imperial University 7, 15 Tôkyô 1933.

Hummel, J. N.: Die Dielektrizitätskonstante der Gesteine. - In ReICH, H. \& ZwERgER, R. v. Taschenb. d. angew. Geophysik. S. 99-112, Leipzig 1943.

Johannson, S.: Neue Experimentaluntersuchungen über die aggregierende Wirkung des Frostes auf dem Erdboden. - Kolloidchem. Beihefte 24, 1932.

JuNG, Erhard: Neue Experimental Untersuchungen über die aggregierende Wirkung des Frostes auf den Boden. Kolloidchem. Beihefte 32, 1931. - -

Weitere Beiträge zur aggregierenden Einwirkung des Frostes auf den Erdboden. Zeitschr. für Pflanzenernährung, Düngung u. Bodenkunde. A. 24, 1932.

Koknonen, P.: Beobachtungen über die Struktur des Bodenfrostes. - Acta Forestalia Fennica 30 , Nr. 3. Helsingfors 1926. - - Beobachtungen über die durch den Bodenfrost verursachte Hebung der Erdoberfläche und in der Frostschicht befindliche Gegenstände. Maataloustieteellinen Aikakauskirja Nr. 3 Helsinki 1930.

Low, A.: Instability of viscosous fluid motion - Nature 65, London 1925.

Nordenskjöld, O.: Über die Natur der Polarländer. - Geogr. Ztschr. 13, 1907. - - Die Polarwelt und ihre Nachbarländer. - Leipzig u. Berlin 1909.

Poser, H.: Beiträge zur Kenntnis der arktischen Bodenformen. - Geol. Rundschau 22, 1921. - Das Problem des Strukturbodens. - Ebendort 24, 1933. - - Bemerkungen zum Strukturbodenproblem. - Cbl. f. Min. usw. 1934, Abt. B.

Romanowskx, V.: Application de la théorie convective aux terrains polygonaux. - Revue d. Geogr. phys. et de Geol. dynam. 12, 1939.

Romanowsky, V. \& Cailleux, A.: Sols polygonaux et fentes de dessication - Bull. Soc. Géol. France (5) 12, 1942.

Schenk, E.: Solifluktion. - Z. deutsch. geol. Ges. 105, 1954. - - Die Mechanik der periglazialen Strukturböden. - Erscheint als Abhandl. des Hess. Landesamtes f. Bodenforsch. 1955.

Sernander, R.: Flytjord i svenska fjälltrakter. - Geol. Fören. Förhandl. 2\%, 1905.

Sörensen, Th.: Bodenformen und Pflanzendecke in Nordostgrönland. - Medd. om Grönland 93, Kobenhavn 1935.

Steche, H.: Beiträge zur Frage der Strukturböden. - Ber. ü. d. Verhdl. d. Sächs. Akad. d. Wissensch. Leipzig 85, 1933.

TABer, St. M.: Frost Heaving. - Journal of Geol. 37, 1929. - - The Mechanics of Frost Heaving. - Ebenda 38, 1930. - - Intensive Frost Action along Lake Shores. - Amer. Journ. of Science 248, 1950.

Troll, C.: Sturkturboden, Solifluktion u. Frostklimate der Erde. - Geol. Rdsch. 34, 1944. - Die Formen der Solifluktion und die periglaziale Bodenabtragung. - Erdkunde 1, 1947.

Vageler, P.: Der Kationen- und Wasserhaushalt des Mineralbodens. - Berlin 1932.

Zunker, F.: Das Verhalten des Bodens zum Wasser. - Hdb. der Bodenlehre VI. Berlin 1930.

Manuskr. eingeg. 7. 6. 1955.

Anschrift des Verf.: Dr. E. Schenk, Gießen, Professorenweg 6. 